\title{
Supplementary material for: Deep learning to accelerate scatterer-to-field mapping for inverse design of dielectric metasurfaces
}

\author{
Maksym V. Zhelyeznyakov, ${ }^{\dagger}$ Steve Brunton, ${ }^{\ddagger}$ and Arka Majumdar*, ${ }^{*}, \uparrow$ \\ $\dagger$ Department of Electrical and Computer Engineering, Seattle, Washington, 98195, USA \\ $\ddagger$ Department of Mechanical Engineering, University of Washington, WA 98195, USA \\ \Department of Physics, Seattle, Washington 98195, USA \\ E-mail: arka@uw.edu
}

\section{Definition of efficiency metrics for the designed devices.}

The standard definition of efficiency for a metasurface lens with a given focal length $f$ is:

$$
\begin{aligned}
\eta & =\frac{\iint_{\Omega} \mathcal{E}^{*}(x, y, z=f) \mathcal{E}^{*}(x, y, z=f) d x d y}{\iint_{x, y} \mathcal{E}^{*}(x, y, z=0) \mathcal{E}^{*}(x, y, z=0) d x d y} \\
\Omega & :=x^{2}+y^{2}<(3 \times F W H M)^{2}
\end{aligned}
$$

where $\Omega$ is the surface around the focal spot which we integrate over, and FWHM is the full width half maximum of a Gaussian fitted to the focal spot.

We quantify the functionality of the annular metasurface as the ratio between the power confined in the annulus to the power confined in the center of the annulus. More formally 
we define $\eta_{\circ}$ as:

$$
\begin{aligned}
\eta_{\circ} & =\frac{\iint_{\Omega_{\circ}} \mathcal{E}^{*}(x, y, z=F) \mathcal{E}^{*}(x, y, z=F) d x d y}{\iint_{\Omega} \mathcal{E}^{*}(x, y, z=F) \mathcal{E}^{*}(x, y, z=F) d x d y} \\
\Omega_{\circ} & :=\left(r_{\circ}+\delta r\right)^{2}<x^{2}+y^{2}<\left(r_{\circ}+\delta r\right)^{2} \\
\Omega_{t} & :=x^{2}+y^{2}<\delta r^{2}
\end{aligned}
$$

Here, $\Omega_{\circ}$ is the surface representing the annulus and $\Omega_{t}$ is the surface representing the center of the annulus. $r_{\circ}$ is the radius of the annulus, defined in the optimization procedure as $1.5 \mu \mathrm{m} . \quad \delta r$ is the thickness over which we integrate, which we define as $\delta r=\frac{1}{2} \mathrm{FWHM}$ calculated for the $\lambda=633 \mathrm{~nm}$ case. $\eta_{\text {o }}$ was found to be equal to 58.47. All integrals are taken over the $\lambda=400 \mathrm{~nm}$ field. Another possible metric of interest would be the fraction of power contained inside the surface $\Omega_{t}$, but in the $\lambda=633 \mathrm{~nm}$ case. We calculate this metric by switching $\Omega$ for $\Omega_{t}$ in Eq. 1. This metric gives an efficiency of $10.55 \%$.

\section{Deep neural network model and training}

Throughout this work, we assumed that the majority of the nearfield coupling is accounted for by incorporating scattering from nearby pillars only. To test this, we created separate DNN models with single scatterer radius (1x1 block), nearby neighbors (3x3 block), as well as second order $(5 \times 5$ block $)$ and third order neighbors $(7 \times 7$ block) as inputs. Fig. S1a summarizes our results. The relative error between the predicted fields and the test field set is largest when only a single scatterer radius is included in the input. When the nearest neighbors are incorporated, the relative error drops significantly. Perhaps somewhat unex-

pectedly, including second and third order nearest neighbors increases the relative error in our test data set. This can be interpreted as the models over-fitting the fields to a larger number of inputs, and could be fixed by adding more data to the training. 

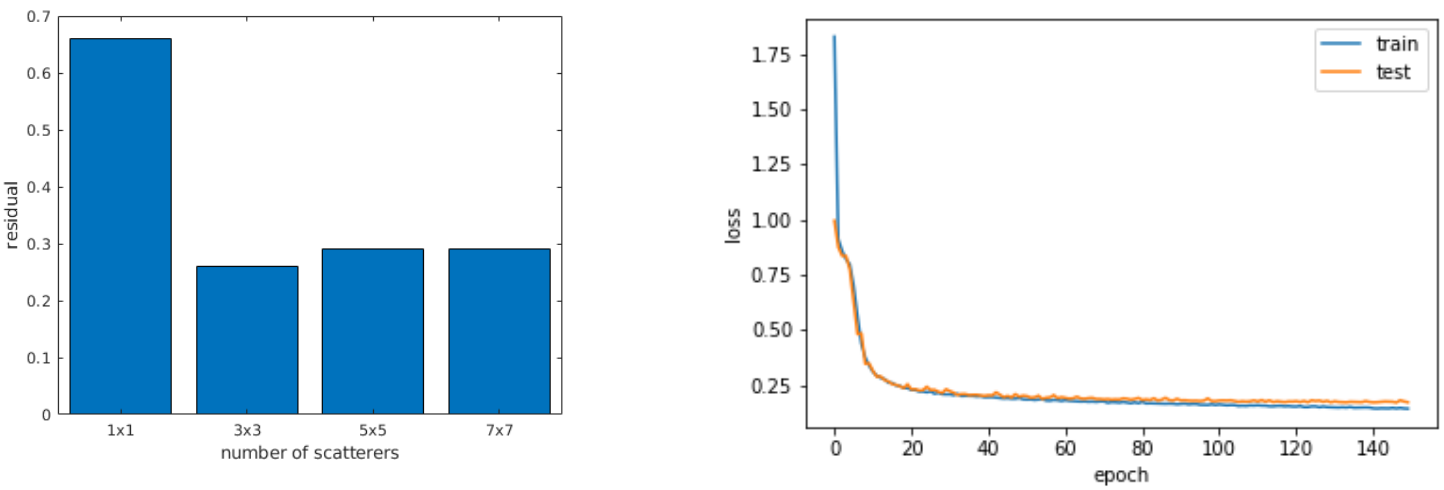

(a) Model accuracy vs number of input features

(b) DNN loss

Figure S1: a. Shows the model accuracy as number of features increases by including no nearby neighbors (1x1 block), nearby neighbors only (3x3 block), second order (5x5 block) and 3rd order (7x7) block nearest neighbors. b. Plot of the DNN objective function as a function of training epoch.

Fig. S1b shows the objective loss of the DNN model with $3 \times 3$ pillar radii as inputs vs the training epoch. Since around epoch 50, the test data set loss starts to diverge from the train data set loss, we use this point as our training cut-off to avoid overfitting. Fig S2 shows the histograms of the field errors predicted from a set of radii by the DNN and the linear models. The error here is defined by:

$$
\left\|E_{F D T D}\left(x_{i}\right)-E_{\text {pred }}\left(x_{i}\right)\right\|_{2}^{2}
$$

The histogram is tighter for the DNN model, thus showing it indeed performs better than the linear model we used. 


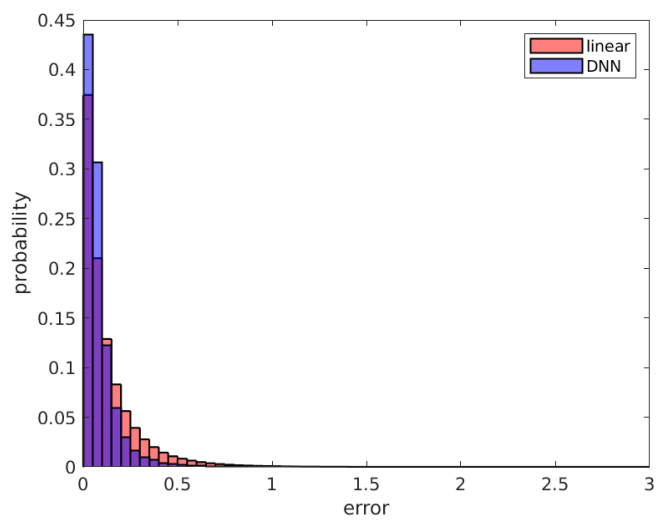

Figure S2: Error histograms of the DNN and Linear models. $\mathrm{Y}$ axis is normalized to the probability of finding a certain error value

\section{Inverse design with the DNN model}

To inverse design both of the devices outlined in the main paper, we use the DNN model as our forward simulation method to compute the nearfield response of the device. Then the nearfields are propagated to the focal plane of interest using the angular spectrum method implemented in TensorFlow. The final fields were used to compute the figures of merit for both of the devices.
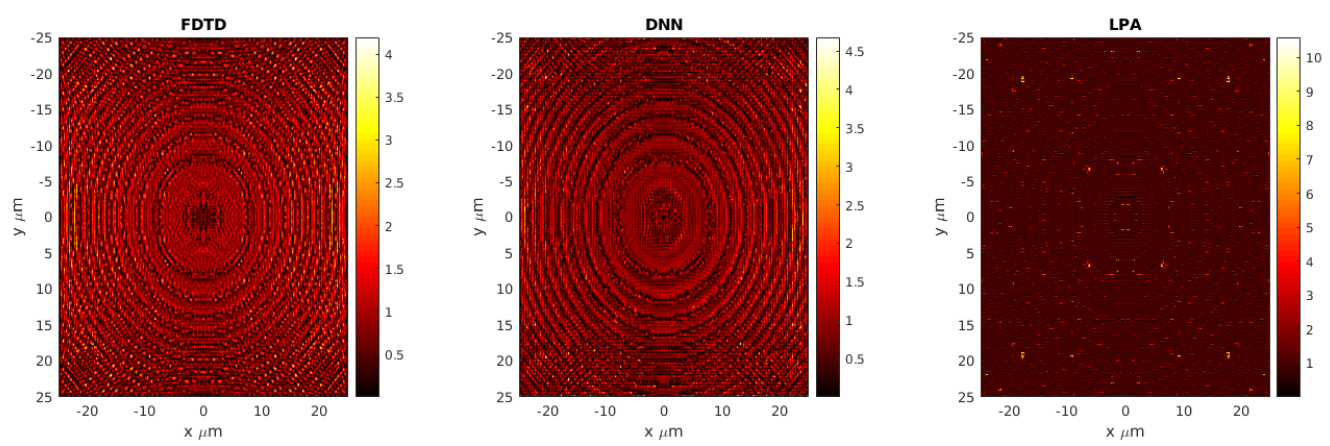

Figure S3: Nearfields for the $f=50 \mu \mathrm{m}$ lens computed with Lumerical FDTD (left), the DNN model (middle), and using RCWA under the local phase approximation (right).

In the case of the wavelength-multiplexed device, we used a forward designed lens that focuses light at focal length $f=50 \mu \mathrm{m}$ as the initial condition. Fig. S3 shows the nearfields of the initial condition computed with three different methods: full FDTD simulation, our DNN model, and RCWA stitching under the LPA. The fields predicted by our method are 
much more similar to FDTD than those predicted with LPA.

Fig. S4 summarizes the the design of the annular device. The left-most figure is the initial condition of the device (a $50 \mu \mathrm{m}$ focal length lens), the second shows the resulting, optimized device, and the third shows the difference between the two. On average, the mean absolute difference between the initial device and the final device is $29.5 \mathrm{~nm}$ with a standard deviation of $24.9 \mathrm{~nm}$.
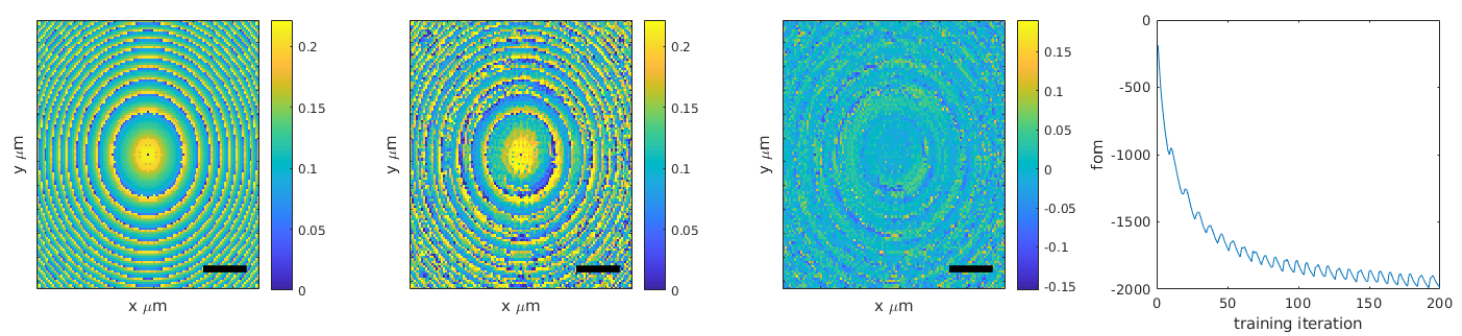

Figure S4: From left to right we show: the initial device, the final designed device, the difference in radii between the initial device and the final device, and the figure of merit during optimization. The scale bars correspond to $10 \mu \mathrm{m}$.

We terminate the optimization after 150 iterations, since the FOM improves only marginally after this point. The final value of the FOM is $-1.99 \times 10^{3}$. The final FOM calculated via FDTD is -788 .

Similarly, the EDOF device was designed by using a forward designed lens that focuses light at $f=100 \mu \mathrm{m}$ as the initial condition. Fig. S5 shows the nearfields calculated with the different methods. Fig. S6 summarizes the optimization of the device.

The final FOM value we calculate for the EDOF lens with our method is -48 , vs the FDTD method -24 . It is clear from the differences in the FOM's between FDTD and our method that our method does not capture all of the physics of the system. However, as shown from the farfields in Figs. S7a and S7b, this is still sufficient to perform inverse design of metasurfaces with alternate functionalities that are impossible to generate with forward design only. 

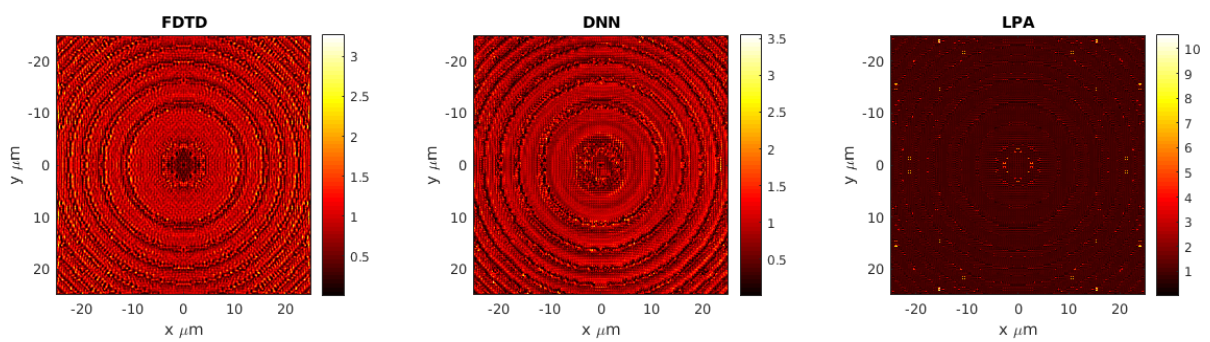

Figure S5: Nearfields for the $f=100 \mu m$ lens computed with Lumerical FDTD (left), the DNN model (middle), and using RCWA under the local phase approximation (right).
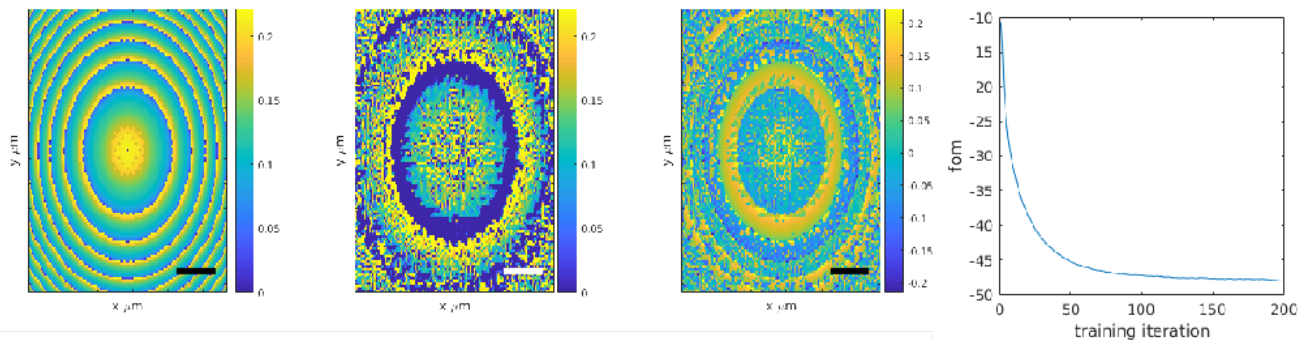

Figure S6: From left to right we show: the initial device, the final designed device, the difference in radii between the initial device and the final device, and the figure of merit during optimization. The scale bars are $10 \mu \mathrm{m}$

\section{Model performance at different wavelengths}

We tested the DNN model's performance 10nm away from its operating wavelength $\lambda=$ $643 \mathrm{~nm}$, and calculated the relative error on the test data set to be $\sim 0.5$. Then we inverse designed the same wavelength switched device as outlined in the paper, at $\lambda=390 \mathrm{~nm}$ for the annular beam and $\lambda=643 \mathrm{~nm}$ for the focal spot. Fig. S8 shows the performance of this device. Note that even with a relative error that is twice than that of the original model, its robust enough for inverse design. 

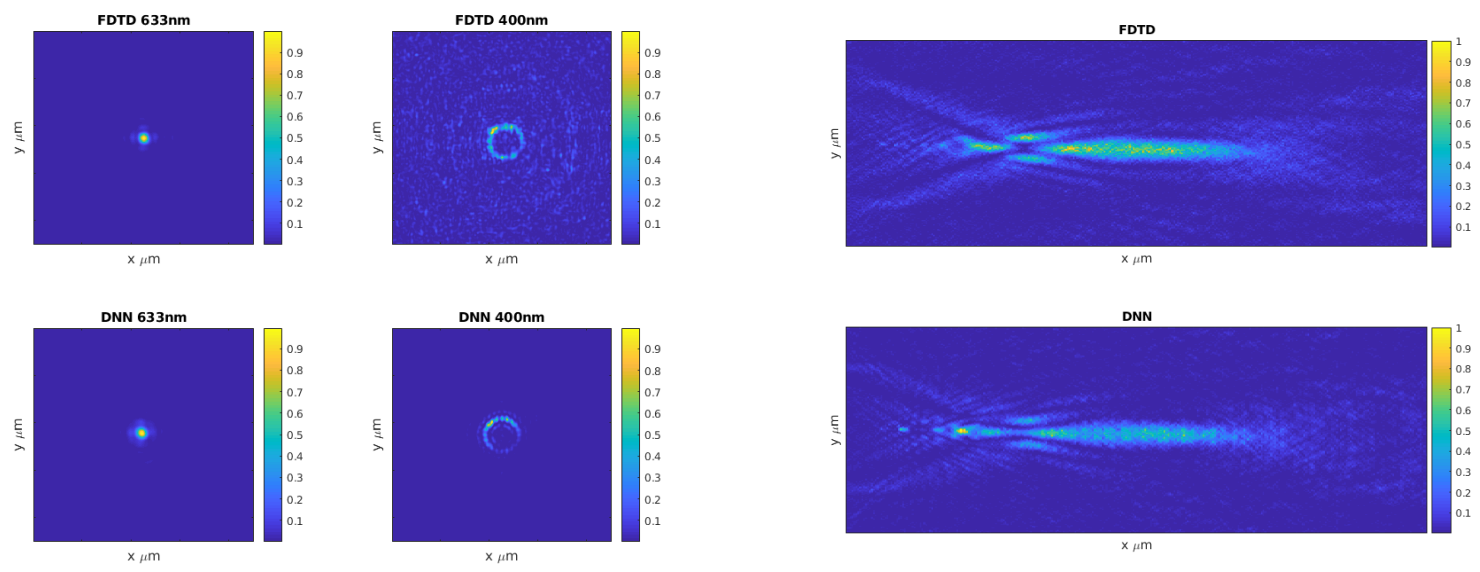

(a) Wavelength switched device

(b) EDOF device

Figure S7: a. Farfields of wavelength switched device and b EDOF device. Top is the field calculated via FDTD and bottom is the field calculated using our method
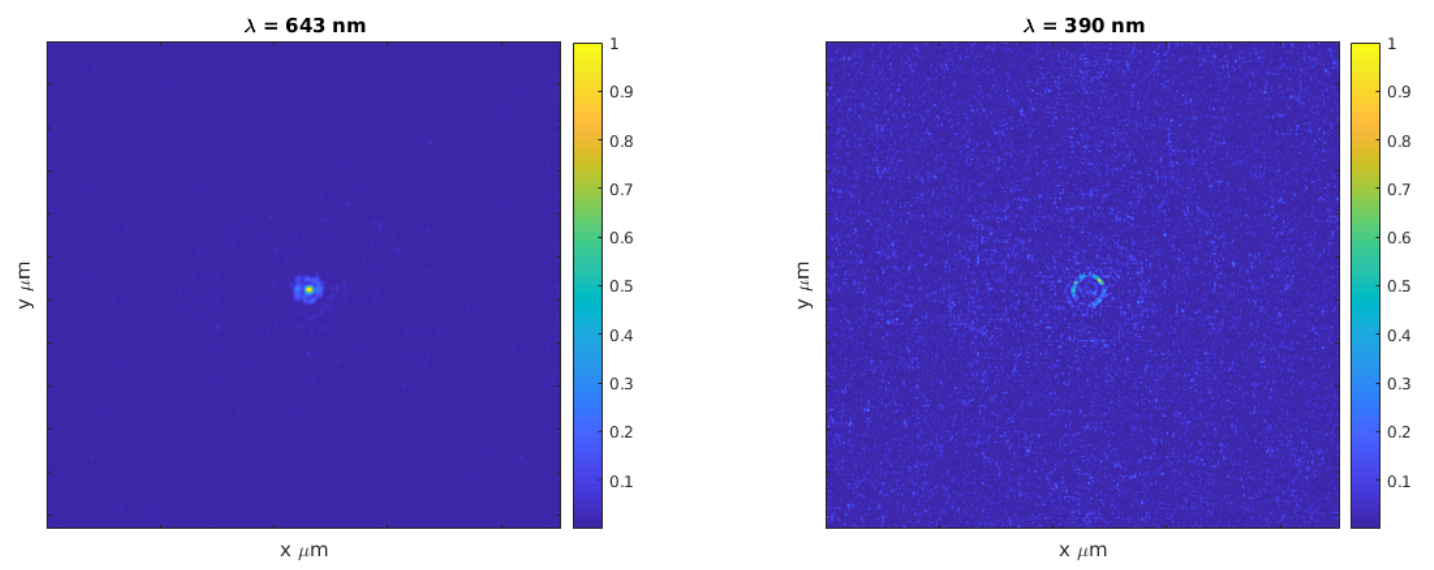

Figure S8: inverse designed wavelength switched device at $\lambda=643 \mathrm{~nm}$ and $\lambda=390 \mathrm{~nm}$ 